\title{
The Role of Diversity in Persistence Aggregation
}

\author{
Roy Cerqueti \\ University of Macerata, Faculty of Economics \\ Department of Economic and Financial Institutions \\ Via Crescimbeni, 20 - 62100 - Macerata, Italy \\ Tel.: +390733 2583246; Fax: +390733 2583205 \\ Email: roy.cerqueti@unimc.it

\section{Giulia Rotundo} \\ University of Tuscia, Faculty of Economics \\ Via del Paradiso, 47 - 01100 - Viterbo, Italy \\ Tel.: +390761357727; Fax: +390761357707 \\ Email: giulia.rotundo@uniroma1.it
}

September 15, 2011

\begin{abstract}
This paper deals with the theoretical analysis of the long-term memory property of time series generated by the aggregation of heterogeneous terms. The diversity is captured by the different features regarding the persistence of each component. It is shown that the memory of the aggregation is driven by the one related to some key components. The argument is carried out by developing an equilibrium model for asset prices in a financial market with heterogeneous agents.
\end{abstract}

\section{Introduction}

The evolution of an economic system is strongly related to the agents populating the system itself. In this regard, it is worth to focus attention to the important role played by the diversity between units.

The analysis if the diversity have become a remarkable aspect of the decision theory for what concerning the selection of multiple elements belonging to different families of candidates (Yager, 2010). 
In some other contexts, diversity rules the connection among heterogeneous agents to share information and collaborate or compete (Gifford and Agah, 2009). In this respect, the diversity may also be an indicator of the performance of the strategies in a dynamic optimization framework (Pelta et al., 2009).

In this paper, we deal with a complex multi-agent system apt to implement the theoretical analysis of the persistence properties of a financial time series. More precisely, we investigate the presence of long memory in the time series of the price of an asset traded in a financial market. The concept of diversity introduced in our model mirrors in the heterogeneity of the agents. In this respect, agents are heterogeneous in the rule adopted to evaluate the future realizations of the asset price and in the way to implement such a rule. The former aspect captures the subjectivity of the beliefs, while the latter takes into account how the agents physically trade, with particular reference to the technical analysis of the market.

The concept of long memory is raised in time series empirical analysis in terms of the persistence of observed autocorrelations. The long memory property is fulfilled by a time series when the autocorrelation decays hyperbolically as the time lag increases. This statistical feature is, therefore, strongly related to the long-run predictability of the phenomenon.

Long memory models were first introduced into the physical sciences in 1950, when some research into applied statistics reported instances of long memory within hydrologic and climatologic data. The earliest studies in this field were carried out by Hurst (1951, 1957), Mandelbrot and Wallis (1968), Mandelbrot (1972), and McLeod and Hipel (1978) among others.

In recent years, quantitative studies of financial markets have shown the persistence properties of the financial time series. In this respect, the long memory of the volatility has been evidenced through the analysis of various speculative returns (Ding and Granger, 1996a and Bollerslev and Mikkelsen, 1996) in power transformations of foreign exchange rate returns (Ding and Granger, 1996b) and also in stock price time series (Ausloos and Ivanova, (1999) and Vandewalle and Ausloos, (1998)). For what concerns the persistence of the prices, this property has been tackled by Wei and Leuthold (2000) in the context of the agricultural futures, while Lo (1991) and Cheung and Lai (1993) focus on the evidence of long memory in certain stock prices and analyze also the gold market returns. Fung et al. (1994) show no consistent pattern of persistence in S\&P 500 index futures prices.

In this paper, a theoretical structural microeconomic model is constructed and developed. We provide the mathematical analysis of the exact relationship between the model parameters which support the presence of long memory at the aggregate level.

We proceed by adopting the approach of the agent-based structural model of Kirman and Teyssiere (2002). The market is populated by heterogeneous agents, and their aggregation affects the structure of the asset price dynamics. In particular, each agent carries out price forecasts using a short term approach, but collective behavior can exhibit long-memory property. In this context, we extend 
some existing results (see Zaffaroni 2004, 2007a, 2007b) about long-memory property arising due to the aggregation of micro units, by enlarging the class of probability densities of agents' parameters. As already stated above, the concept of diversity contained in our model pertains to agents' heterogeneity. In this respect, two cases of heterogeneity are developed:

- The first case concerns agents wearing two different hats at the same time: the asset price forecasts are driven by a fundamentalist and a chartist one. An agent is fundamentalist if she/he believes that the price of an asset is determined by its fundamental value. In contrast, chartists carry out technical analysis of the market and do not take the fundamentals into account (see Brock and Hommes (1998) for a financial market populated by fundamentalist and chartist investors).

- The second case relies to agents performing different types of technical analysis of the market. In this respect, we highlight the distinction between impulsive traders and long period investors. The former type of agents takes position in a market for a very short horizon, while the trading strategies of the latter type are performed in a long time horizon.

The rest of the paper is organized as follows: Section 2 introduces the economic model; Section 3 contains the analysis of the long memory property, with a particular reference to the diversity among agents. Last section concludes and outlines some further research lines. The proofs of the main results are relegated to the Appendix.

\section{The model}

The basic features of the market model we are going to set up, are the existence of two groups of agents, with heterogeneity inside each group.

Let us consider a market with $N$ agents who can invest either in a risk-free or in a risky asset. The risk free bond has a constant interest rate $r \in(0,1)$.

Let $P_{t}$ the price of the risky asset and $P_{i, t}$ the estimate of it carried out by the agent $i$ at time $t$. The change of the price at time $t+1$ forecast by the $i$-th agent, conditioned to her/his information at time $t, I_{i, t}$, is given by $\Delta P_{i, t+1} \mid I_{i, t}$.

We assume that the market is not efficient, i.e. we can write the following relationship:

$$
\mathbb{E}\left(P_{t+1} \mid I_{t}\right)=\Delta P_{t+1} \mid I_{t}+P_{t}
$$

where $\mathbb{E}$ is the expected value operator and $I_{t}$ is the information available up to time $t$.

The behavior of the investors is due to analysis of the market data (using a typical chartist approach) and to the exploration of the behavior of market fundamentals (using a fundamentalist approach). Moreover, the forecasts are influenced by an error term, common to all the agents:

$$
\left(\Delta P_{i, t+1} \mid I_{i, t}\right)=\left(\Delta P_{i, t+1}^{c} \mid I_{i, t}\right)+\left(\Delta P_{i, t+1}^{f} \mid I_{i, t}\right)+u_{t},
$$


where $\left(\Delta P_{i, t+1}^{c} \mid I_{i, t}\right)$ is the contribution of the chartist approach, $\left(\Delta P_{i, t+1}^{f} \mid I_{i, t}\right)$ is associated to the fundamentalist point of view and $u_{t}$ is a stochastic term representing an error in forecasts, i.e. $u_{t}$ is i.i.d. with mean 0 and variance $\sigma_{u}^{2}$.

As a first step, we assume without loss of generality ${ }^{1}$ that all the agents have the same weight in the market and that the price $P_{t}$ of the asset in the market at time $t$ is given by the mean of the asset price of each agent at the same time. So we can write

$$
P_{t}=\frac{1}{N} \sum_{i=1}^{N} P_{i, t} .
$$

Equation (3) is a type of market clearing price condition.

We now describe the price formation mechanism of the agents.

The chartists glean information from the time series of market prices. The $i$-th agent's price change forecast is assumed to be given by the following linear combination:

$$
\Delta P_{i, t+1}^{c} \mid I_{i, t}=\alpha_{i}^{(1)}\left(P_{i, t}-P_{i, t-1}\right)+\alpha_{i}^{(2)} P_{t},
$$

with $\alpha_{i}^{(1)}, \alpha_{i}^{(2)} \in[0,+\infty), \forall i=1, \ldots, N$. Formula (4) encapsulates the idea of a stochastic relationship providing the estimated change in prices by relying on a linear combination of the two previous price forecasts, adjusted to the actual market price obtained at the relative time.

The fundamentalist approach takes the analysis made by the investors about market fundamental value into account.

The fundamental variables $\bar{P}_{i, t}$ can be described by the following random walk:

$$
\bar{P}_{i, t}=\bar{P}_{i, t-1}+\epsilon_{t}, \quad \epsilon_{t} \sim N\left(0, \sigma_{\epsilon}^{2}\right) .
$$

The fundamental prices observed by the agent $i$ at time $t, \tilde{P}_{i, t}$, are assumed to be biased by a stochastic error:

$$
\tilde{P}_{i, t}=\bar{P}_{i, t}+\bar{\alpha}_{i, t},
$$

with $\bar{\alpha}_{i, t}=\beta_{i} P_{t}$, where the $n$-ple $\left(\beta_{1}, \ldots, \beta_{N}\right)$ is drawn by sampling from the cartesian product $(1-\xi, 1+\xi)^{N}, \xi>0$, equipped with the relative product probability measure. The definition of $\bar{\alpha}_{i, t}$ takes into account the fact that the error in estimating depends on the adjustment performed by each agent of the market price. More precisely, the observation of the fundamental prices is affected by the subjective opinion of the agents about the influence of the market price on the fundamental. If $\beta_{i}>1$, then agent $i$ guesses that the market price is responsible for an overestimate of fundamental prices. Otherwise, the converse situation applies.

Moreover, the forecasts of the fundamentalist agents are based on fundamental prices and their forecasts about market prices at the previous data. So we can write

$$
\Delta P_{i, t+1}^{f} \mid I_{i, t}=\nu\left(\tilde{P}_{i, t}-P_{t}\right),
$$

\footnotetext{
${ }^{1}$ It is easy to prove that our arguments continue to hold, also when the agents have different sizes in the market (see Cerqueti and Rotundo, 2010).
} 
with $\nu \in \mathbb{R}$. Thus

$$
\Delta P_{i, t+1}^{f} \mid I_{i, t}=\nu \bar{P}_{i, t}+\nu\left(\beta_{i}-1\right) P_{t} .
$$

Remark 1. By comparing (4) and (7), it must be $\alpha_{i}^{(2)}=\nu\left(\beta_{i}-1\right)$. We state this condition for the remaining part of the paper.

Let us define $d_{i, t}$ to be the demand of the risky asset of the agent $i$ at the date $t$.

The estimated wealth of the agent $i$ at time $t+1$ is given by $W_{i, t+1}$, and it is given by:

$$
W_{i, t+1}=\left(1+\frac{P_{i, t+1}-P_{i, t}}{P_{i, t}}\right) P_{i, t} d_{i, t}+\left(W_{i, t}-P_{i, t} d_{i, t}\right)(1+r) .
$$

By (8), the expression of $W_{i, t+1}$ can be rewritten as:

$$
W_{i, t+1}=\Delta P_{i, t+1} d_{i, t}+W_{i, t}(1+r)-r P_{i, t} d_{i, t} .
$$

Each agent $i$ at time $t$ optimizes the mean-variance utility function

$$
U\left(W_{i, t+1} \mid I_{i, t}\right)=\mathbb{E}\left(W_{i, t+1} \mid I_{i, t}\right)-\mu \mathbb{V}\left(W_{i, t+1} \mid I_{i, t}\right),
$$

where $\mathbb{V}$ is the usual variance operator, and thus:

$$
\mathbb{E}\left(W_{i, t+1} \mid I_{i, t}\right)=\left(\Delta P_{i, t+1} \mid I_{i, t}\right) d_{i, t}+W_{i, t}(1+r)-r P_{i, t} d_{i, t}
$$

and

$$
\mathbb{V}\left(W_{i, t+1} \mid I_{i, t}\right)=\mathbb{V}\left(P_{i, t+1} \mid I_{i, t}\right)\left(d_{i, t}\right)^{2} .
$$

Each agent $i$ maximizes her/his expected utility with respect to her/his demand $d_{i, t}$, conditioned to her/his information at the date $t$. For each agent $i$ the first order condition is

$$
\left(\Delta P_{i, t+1} \mid I_{i, t}\right)-r P_{i, t}-2 \mu \mathbb{V}\left[\left(P_{i, t+1} \mid I_{i, t}\right)\right] d_{i, t}=0,
$$

By the first order conditions we obtain

$$
d_{i, t}=b_{i, t} P_{i, t}+g_{i, t}\left(\Delta P_{i, t+1} \mid I_{i, t}\right)
$$

with

$$
b_{i, t}=\frac{-r}{2 \mu \mathbb{V}\left(\left(P_{i, t+1} \mid I_{i, t}\right)\right)} ; \quad g_{i, t}=\frac{1}{2 \mu \mathbb{V}\left(\left(P_{i, t+1} \mid I_{i, t}\right)\right)} .
$$

Let $X_{i, t}$ be the supply function at time $t$ for the agent $i$. We have the following equilibrium relation:

$$
X_{i, t}=b_{i, t} P_{i, t}+g_{i, t}\left(\Delta P_{i, t+1} \mid I_{i, t}\right) .
$$

Let us denote

$$
\gamma_{i, t}=\frac{X_{i, t}}{b_{i, t}}, \quad c=-\frac{1}{r}=\frac{g_{i, t}}{b_{i, t}}, \quad \lambda_{i}:=\frac{-c \alpha_{i}^{(2)}}{1+c \alpha_{i}^{(1)}} .
$$


By (2), (4), (7) and (10) we get:

$$
P_{i, t}=\frac{1}{1+c} \cdot \frac{1-\lambda_{i}}{1-\lambda_{i} L}\left\{\gamma_{i, t}-c \nu \bar{P}_{i, t}-u_{t}\right\},
$$

where $L$ is the backward time operator, i.e. $L P_{i, t}=P_{i, t-1}$.

Condition (3) and equation (12) allow us to write the market price as

$$
P_{t}=\frac{1}{N} \sum_{i=1}^{N}\left[\frac{1}{1+c} \cdot \frac{1-\lambda_{i}}{1-\lambda_{i} L}\left\{\gamma_{i, t}-c \nu \bar{P}_{i, t}-u_{t}\right\}\right] .
$$

The parameter $\lambda_{i}$ is particularly relevant in describing the heterogeneity of the agents. Indeed, it provides information on the technical analysis of the market performed by the $i$-th agent. As we will see below, the $\lambda$ 's play a central role in determining the persistence properties of the price.

\section{Diversity and long term memory}

This section shows the long-term memory property of market price time series. In particular, we focus on the theoretical conditions on the parameters distribution and on the stochastic processes that are needed for long memory.

In order to proceed, the following technical assumption is needed:

Assumption 2. $\alpha_{i}^{(2)}<r$.

The relation between the indices $i$ and $t$ in defining the process $\gamma_{i, t}$ is outlined in the following Assumption.

Assumption 3. There exist $N$ random variables $w_{1}, \ldots, w_{N}$ and a stochastic process $z_{t}$, independent on $u_{t}$, such that:

- $\mathbb{E}\left[w_{j}\right]=\bar{\omega} \in \mathbb{R}$, for each $j=1, \ldots, N$;

- $w_{i}$ is independent on $\lambda_{i}$, for each $i=1, \ldots, N$;

- $z_{t}$ are i.i.d., with mean 0 and variance $\sigma^{2}$;

- for each $i=1, \ldots, N$ and $t \geq 0$, it results $\gamma_{i, t}=z_{t} \cdot w_{i}$.

Assumption 3 states that in our model the excess of demand compensates, on average, the excess of supply. The diversity in our model mirrors in the distributional hypotheses on the agent-based random variables $\lambda$ 's. As already stressed above, the parameter $\lambda_{i}$ provides a description of the forecast rule used by the $i$-th agent when wearing a chartist hat. In this respect, homogeneity means that $\lambda_{i}$ are identically distributed, while heterogeneity holds otherwise.

In determining the distributional hypothesis on the $\lambda$ 's, we basically take into account two types of investors: impulsive traders and long run traders. The former type of agents performs an analysis 
of the market, following a chartist approach, only in rare situation. The latter type of agents deals with a technical analysis of the market continuously in time.

We initially analyze homogeneity among agents, and then move to heterogeneity.

The first result concerns the case of a very general two-parameter distribution, able to describe several types of agents as the value of the parameters varies. For the proof, see the Appendix.

Theorem 4. Let us assume that there exists $a, b \in(0,+\infty)$ such that $\lambda_{i}$ are sampled by a $B(a, b)$ distribution, for each $i=1, \ldots, N$.

Then, as $N \rightarrow+\infty$, the long-term memory property for $P_{t}$ holds, with Hurst's exponent $H_{B} \leq 1 / 2$.

As the parameters of the Beta distribution vary, several types of continuous-time traders may be described. Furthermore, the proof of Theorem 4 evidences that the distributional hypothesis on $\lambda$ may be relaxed. The following Corollary states immediately:

Corollary 5. Assume that:

$$
\mathbf{E}\left[\lambda_{i}^{k}\right] \sim O(c) k^{-1-b}+o\left(k^{-1-b}\right) \text { as } k \rightarrow+\infty .
$$

Then, as $N \rightarrow+\infty$, the long-term memory property for $P_{t}$ holds, with Hurst's exponent $H_{B} \leq 1 / 2$.

We now move from homogeneity to agents gathered in several groups. Each group has its own impact on the market and exhibits organized heterogeneity among its components.

By a mathematical perspective, this assumption is equivalent to the study of the aggregate of a mixture of absolute continuous distributions for the parameters $\lambda$ 's.

More precisely, we introduce a group of investors that concentrate their attention in a small set of events, i.e. the behavior of these agents is given by not assuming a position for the most part of the market traffic, and take part heavily in some particular and rare situations. We formalize this kind of behavior by using Dirac measures $\delta_{x}(y)$ as follows:

$$
\delta_{x}(y)=\left\{\begin{array}{cc}
1, & \text { for } x=y \\
0, & \text { for } x \neq y
\end{array}\right.
$$

The proof of next result is contained in the Appendix.

Theorem 6. Consider $b_{1}, \ldots, b_{k} \in(0,+\infty)$ and $A_{1}(N), \ldots, A_{k}(N) \subseteq\{1, \ldots, N\}$ such that $\lambda_{i}$ are sampled by $B\left(a, b_{j}\right)$ distribution, for each $i \in A_{j}(N), j=1, \ldots, k$.

Moreover, consider $d_{k+1}, \ldots, d_{n} \in(0,1)$ and $A_{k+1}(N), \ldots, A_{n}(N) \subseteq\{1, \ldots, N\}$ such that $\lambda_{i} \sim \delta_{d_{i}}$, for each $i \in A_{j}(N), j=k+1, \ldots, n$.

Assume that there exists $p_{j} \in(0,1)$ such that

$$
\lim _{N \rightarrow+\infty} \frac{\operatorname{card} A_{j}(N)}{N}=p_{j}, \quad \forall j=1, \ldots, n .
$$

Furthermore, assume that $\lambda_{i}$ are sampled by independent random variables.

Then, as $N \rightarrow+\infty, P_{t}$ has the long term memory, with Hurst's exponent $H_{D} \leq 1 / 2$. 


\section{Conclusions and further developments}

In this work we analyze the role of the diversity of agents in exploring long term memory of financial time series. The agents are supposed to drive actively the price formation of an asset, and heterogeneity mirrors in the way to make forecasts (chartist and fundamentalist) and in the way to technically analyze the market (distribution of the parameters $\lambda$ 's).

We extend some results present in the literature about the arise of the long memory property due to the aggregation of independent micro units.

We prove that the persistence of the price process cannot be explained by the contributions due to spot traders. This finding is completely compatible with the evidence: the occurrence of an impulsive phenomenon cannot be responsible for long-run equilibrium properties.

This model has a natural extension in the study of the persistence properties of the price dynamics in presence of correlation among agents. In this respect, we aim at entering the endogenous correlation structure of the agents, by measuring the impact of a hierarchical structure in price persistence as well as the presence of a contagion effect.

\section{References}

[1] Ausloos, M., Ivanova, K., (1999). Low $q$-moment multifractal analysis of gold price, Dow Jones Industrial Average and BGL-USD exchange rate. European Physical Journal, 8, 665-669.

[2] Bollerslev, T., Mikkelsen, H.O. (1996). Modelling and pricing long memory in stock market volatility. Journal of Econometrics, 73, 151-184.

[3] Cerqueti, R., Rotundo, G., (2010). Memory Property in Heterogeneously Populated Markets. In: S. Greco, R.A. Marques Pereira, M. Squillante, R.R. Yager and J. Kacprzyk Eds., Preferences And Decisions - Studies in Fuzziness and Soft Computing, Volume 257/2010, Springer-Verlag, $53-67$.

[4] Cheung, Y.W., Lai, K.S., (1993). A fractional cointegration analysis of purchasing power parity. Journal of Business and Economic Statistics, 11, 103-12.

[5] Ding, Z., Granger, C.W.J., (1996a). Varieties of long memory models. Journal of Econometrics, $73,61-77$.

[6] Ding, Z., Granger, C.W.J., (1996b). Modelling volatility persistence of speculative returns: a new approach. Journal of Econometrics, 73, 185-215.

[7] Fung, H.K., Lai, S., Lai, M., (1994). Fractal Structure in Currency Futures Price Dynamics. The Journal of Futures Markets, 169-181. 
[8] Gifford, C.M., Agah, A., (2009). Sharing in Teams of Heterogeneous, Collaborative Learning Agents. International Journal of Intelligent Systems, 24, 173-200.

[9] Granger, C.W.J., (1980). Long memory relationships and the aggregation of dynamic models. Journal of Econometrics, 14, 227-238.

[10] Hurst, H., (1951). Long Term Storage Capacity of Reservoirs. Transactions of the American Society of Civil Engineers, 116, 770-799.

[11] Hurst, H.E., (1957). A suggested statistical model of some time seris which occur in nature. Nature 180, 494.

[12] Kirman, A.P., Teyssiére, G., (2002). Microeconomic models for long-memory in the volatility of financial time series. Studies in Nonlinear Dynamics and Econometrics, 5, 281-302.

[13] Lo, Andrew W., (1991). Long memory in stock market prices. Econometrica 59, 1279-1313.

[14] Mandelbrot, B.B., (1972). Possible Refinements of the Lognormal Hypothesis Concerning the Distribution of Energy Dissipation in Intermittent Turbulence. In: Rosenblatt M and Van Atta C. (Eds.) Statistical Models and Turbulence, New York, Springer-Verlag.

[15] Mandelbrot, B.B., Wallis, J.R., (1968). Noah, Joseph, and Operational Hydrology. Water Resources Research, 4(3), p 909-918.

[16] McLeod, A.I., Hippel, A.W., (1978). Preservation of the rescaled adjusted range, 1: a reassessment of the Hurst phenomenon. Water Resources Research, 14, 491-508.

[17] Pelta, D. Cruz, C., Gonzales, J.R., (2009). A Study on Diversity and Cooperation in a Multiagent Strategy for Dynamic Optimization Problems. International Journal of Intelligent Systems, $24,844-861$.

[18] Rangarajan, G., Ding, M., (2000). Integrated approach to the assessment of long range correlation in time series data. Physical Review E 61, no. 5, 4991-5001.

[19] Vandewalle, N., Ausloos, M., (1998). Spareness and Roughness of Foreign Exchange Rates. International Journal of Modern Physics C, 9, 711-720.

[20] Wei, A., Leuthold, R.M., (2000). Agricultural Futures Prices and Long Memory Processes. OFOR Working Paper No. 00.04. Available at SSRN: http://ssrn.com/abstract=229795 or doi:10.2139/ssrn.229795

[21] Yager, R.R., (2010). Including a Diversity Criterion in Decision Making. International Journal of Intelligent Systems, 25, 958-969. 
[22] Zaffaroni, P., (2004). Contemporaneous aggregation of linear dynamic models in large economies. Journal of Econometrics, 120, 75-102.

[23] Zaffaroni, P., (2007a). Memory and aggregation for models of changing volatility. Journal of Econometrics, 136, 237-249.

[24] Zaffaroni, P., (2007b). Contemporaneous aggregation of GARCH processes. Journal of Time Series Analysis, 28, 521-544.

\section{Appendix}

\section{Proof of Theorem 4}

To prove the result, we need to rewrite the process $P_{t}$ as the sum of three components:

$$
P_{t}=\Gamma_{t}^{1}+\Gamma_{t}^{2}+\Gamma_{t}^{3},
$$

where

$$
\left\{\begin{array}{l}
\Gamma_{t}^{1}=\frac{1}{N(1+c)} \sum_{i=1}^{N} \frac{1-\lambda_{i}}{1-\lambda_{i} L} \gamma_{i, t} \\
\Gamma_{t}^{2}=\frac{-c \nu}{N(1+c)} \sum_{i=1}^{N} \frac{1-\lambda_{i}}{1-\lambda_{i} L} \bar{P}_{i, t} \\
\Gamma_{t}^{3}=-\frac{1}{N(1+c)} \sum_{i=1}^{N} \frac{1-\lambda_{i}}{1-\lambda_{i} L} u_{t} .
\end{array}\right.
$$

By definition of the model, the processes $\Gamma$ 's are independent. Hence, we can analyze separately the long term memory property of the $\Gamma$ 's.

Denote as $\lambda$ and $w$ the random identically distributed random variables $\lambda_{i}$ and $w_{j}$. Furthermore, denote as $F$ the joint cumulative distribution function of $(\lambda, w)$ and $F_{\Lambda}$ be the marginal distribution of $\lambda$.

In the limit for $N \rightarrow \infty$ we have

$$
\begin{gathered}
\Gamma_{t}^{1}=\lim _{N \rightarrow+\infty} \frac{1}{N(1+c)} \sum_{i=1}^{N} \frac{1-\lambda_{i}}{1-\lambda_{i} L} \gamma_{i, t}=-\frac{1}{1+c} \int_{0}^{1} \frac{1-\lambda}{1-\lambda L} w z_{t} d F(\lambda, w)= \\
=-\frac{1}{1+c} \bar{\omega} \int_{0}^{1} \frac{1-\lambda}{1-\lambda L} z_{t} d F_{\Lambda}(\lambda)=-\frac{1}{1+c} \bar{\omega} \int_{0}^{1}(1-\lambda) \sum_{k=0}^{\infty}(\lambda L)^{k} z_{t} d F_{\Lambda}(\lambda)= \\
=-\frac{1}{1+c} \bar{\omega} \sum_{k=0}^{\infty} \int_{0}^{1}(1-\lambda) \lambda^{k} z_{t-k} d F_{\Lambda}(\lambda)=-\frac{1}{1+c} \bar{\omega} \sum_{k=0}^{\infty}\left[\int_{0}^{1}(1-\lambda) \lambda^{k} d F_{\Lambda}(\lambda)\right] z_{t-k}=: \sum_{k=0}^{\infty} a_{k} z_{t-k},
\end{gathered}
$$

where

$$
a_{k} \sim \int_{0}^{1}(1-\lambda) \lambda^{k-1} d F_{\Lambda}(\lambda)=\mathbf{E}\left[\lambda^{k}\right]-\mathbf{E}\left[\lambda^{k+1}\right] .
$$


Since $\lambda \sim B(a, b)$, we have:

$$
a_{k} \sim k^{-b-2} .
$$

Therefore, $\Gamma_{t}^{1}$ faces the same asymptotic behavior of an $I(d)$ process, with $d=-b-1$. Since $b>0$, we have that $\Gamma_{t}^{1}$ can be represented as an integrated process of order $d<-1$. Hence, $\Gamma_{t}^{1}$ does not have the long-term memory property.

For what regards the process $\Gamma_{t}^{3}$, fixed $h>0$, we have

$$
\begin{gathered}
\mathbf{E}\left[\Gamma_{t}^{3} \Gamma_{t-h}^{3}\right]=\mathbf{E}\left[\frac{1}{N} \cdot \frac{-c}{1+c} \sum_{i=1}^{N} \frac{1-\lambda_{i}}{1-\lambda_{i} L} u_{t} \cdot \frac{1}{N} \cdot \frac{-c}{1+c} \sum_{j=1}^{N} \frac{1-\lambda_{j}}{1-\lambda_{j} L} u_{t-h}\right]= \\
=\mathbf{E}\left[\frac{c^{2}}{(1+c)^{2}} \sum_{m=0}^{\infty} \int_{0}^{1}(1-\lambda) \lambda^{m} u_{t-m} \mathrm{~d} F(\lambda) \sum_{l=0}^{\infty} \int_{0}^{1}(1-\mu) \mu^{l} u_{t-h-l} \mathrm{~d} F(\mu)\right]= \\
=\frac{1}{\beta(a, b)} \cdot \frac{c^{2} \sigma_{u}^{2}}{(1+c)^{2}} \sum_{l=0}^{\infty} \int_{0}^{1}(1-\lambda)^{1+b} \lambda^{2 l+h+a-1} \mathrm{~d} \lambda= \\
=\frac{1}{\beta(a, b)} \cdot \frac{\sigma_{u}^{2}}{(1+c)^{2}} \sum_{l=0}^{\infty} \frac{\Gamma(h+a+2 l) \Gamma(b+2)}{\Gamma(h+a+b+2 l+2)} \sim \frac{1}{\beta(a, b)} \cdot \frac{\sigma_{u}^{2}}{(1+c)^{2}} h^{-1-b} .
\end{gathered}
$$

Then, Rangarajan and Ding (2000) assures that: as $N \rightarrow+\infty$, the long-term memory property for $\Gamma_{t}^{3}$ holds, with Hurst's exponent $H_{3}$ as follows:

- $b>1$ implies $H_{3}=1 / 2$ :

- $b \in(0,1)$ and the following equation holds:

$$
\sum_{h=-\infty}^{+\infty} \mathbf{E}\left[\Gamma_{t}^{3} \Gamma_{t-h}^{3}\right]=0
$$

imply $H_{3}=(1-b) / 2$. In this case it results $H_{3}<1 / 2$, and the process $\Gamma_{t}^{3}$ is mean reverting.

Since

$$
\bar{P}_{i, t}=\sum_{j=0}^{t-1} \epsilon_{t-j}+\bar{P}_{i, 0}
$$

then $\bar{P}_{i, t}$ is a stationary process, and the arguments carried out for $\Gamma_{t}^{3}$ can be replicated to state that the long memory property holds for $\Gamma_{t}^{2}$ as $N \rightarrow+\infty$. The Hurst exponent is $H_{2}$.

By Granger (1980), we have that

$$
H_{B}=\max \left\{H_{2}, H_{3}\right\}
$$

\section{Proof of Theorem 6}

The process $P_{t}$ can be disaggregated as follows:

$$
P_{t}=\sum_{j=1}^{k} \Phi_{t}^{j}+\sum_{j=k+1}^{n} \Psi_{t}^{j}
$$


where

$$
\left\{\begin{array}{rlrl}
\Phi_{t}^{j} & =\frac{1}{N} \sum_{i \in A_{j}(N)}\left[\frac{1}{1+c} \cdot \frac{1-\lambda_{i}}{1-\lambda_{i} L}\left\{\gamma_{i, t}-c \nu \bar{P}_{i, t}-u_{t}\right\}\right], & j=1, \ldots, k \\
\Psi_{t}^{j}=\frac{1}{N} \sum_{i \in A_{j}(N)}\left[\frac{1}{1+c} \cdot \frac{1-\lambda_{i}}{1-\lambda_{i} L}\left\{\gamma_{i, t}-c \nu \bar{P}_{i, t}-u_{t}\right\}\right], & j=k+1, \ldots, n .
\end{array}\right.
$$

In order to proceed, we need to study the behavior of the $k$-th moments of the Dirac distribution, with $k \in \mathbb{N}$.

A direct computation gives:

$$
\mathbb{E}\left[\left(\delta_{x}\right)^{k}\right]=\int_{-\infty}^{+\infty} \xi^{k} \delta_{x}(\xi) \mathrm{d} \xi=x^{k} .
$$

Therefore, the terms related to the processes $\Psi$ 's do not contribute to the long memory of the process $P_{t}$.

By Theorem 4, we have that the process $\Phi_{t}^{j}$ has an Hurst exponent $H_{j} \leq 1 / 2$. Since the $\lambda$ 's are independent and by Granger (1980), we obtain that

$$
H_{D}=\max \left\{H_{1}, \ldots, H_{k}\right\} \leq 1 / 2,
$$

and this completes the proof. 